\title{
A hybrid ceramic quadband antenna for handset applications
}

\author{
S P Kingsley ${ }^{1}$, D Iellici ${ }^{1}$, S G O'Keefe ${ }^{2}$, P J Ollitervo ${ }^{1}$, B S Collins $^{1}$, \& S Zhang ${ }^{1}$ \\ ${ }^{1}$ Antenova Ltd., Stow-cum-Quy, Cambridge CB5 9AR, UK \\ ${ }^{2}$ Griffith University, Brisbane, Australia
}

\begin{abstract}
A novel internal quadband antenna for radiotelephone handset applications is presented. The design uses a hybrid of ceramic and conventional antenna technologies and shows good efficiency characteristics. With a volume of $3.5 \mathrm{cc}$, the antenna is smaller than current in-service tri and quad band designs. $A$ method of extending the design for diversity or MIMO applications at the higher frequencies is discussed.
\end{abstract}

Index terms- dielectric antenna, handset antenna, high dielectric ceramics, MIMO antennas.

\section{INTRODUCTION}

Antenova has been developing ceramic-based dielectric technology as a means of building high performance antennas for WLAN and handset applications. The technology behind the designs was presented recently [1]. The main advantages of using ceramics for handsets is that high terminal efficiencies are available and the designs are somewhat more resistant to detuning than conventional internal PIFA antennas because of the high stored energy contained within the dielectric.

Resistance to detuning is important as many modern phones have movable parts (clamshells, flip-phones, sliders, etc.) and the antenna must work efficiently when these phones are both open and closed. Handsets must also work well whether they are held in the hand next to the head or are operated remotely from the user. Finally, in the design phase of a handset when many components are moved around, fewer antenna re-designs are required when the antenna resists detuning and this can save some of the design costs.

The design presented at [1] made use of groundplane removal beneath the ceramic pellet as a means of achieving the required frequency coverage for a quadband antenna ( 824 - $960 \mathrm{MHz}$ and $1710-1990 \mathrm{MHz}$ ). For many types of antenna it can be advantageous to remove part of the ground plane through all the layers of a PCB (PWB) as this can help to improve the bandwidth of the antenna. Unfortunately, many modern mobile telephone handsets have so many components to be fitted on the side opposite the antenna (speakers, headphone sockets, USB connectors, display technology, etc.) that it is preferable not to remove the ground plane, either fully or partially. It is therefore desirable to find a way of designing an antenna for mounting on a PCB, the antenna having the wide bandwidth required for modern mobile telephone handsets, while still retaining a full ground plane beneath the antenna. In this paper we show that it is possible to adopt the alternative strategy of moving the dielectric component of the antenna upwards away from the groundplane instead of sideways away from it.

In general, a dielectric antenna consists of a volume of high dielectric material located on or close to a grounded substrate, with the energy being transferred to and from it by a standard feed mechanism such as a probe, slot, microstrip transmission line, coplanar waveguide. When the dielectric is in contact with the groundplane it can be made to work as a classical dielectric resonator antenna (DRA), see [2,3] for example. DRAs have some excellent properties when the $\varepsilon r$ is high enough, but because they tend to have a single classical resonance with a well-defined internal field structure, they do not usual have the bandwidth required for modern communication systems.

When a dielectric pellet is elevated above the groundplane the E-field has a less well-defined structure leading to a less well-defined frequency response and therefore improved bandwidth. The primary radiating mechanism remains a displacement current flowing within the dielectric material or pellet, as in a DRA.

Dielectrics can be efficient radiators, if the $Q$ of the material is high enough, and they can be made into very effective upper-band antennas. Unfortunately, current lowband versions are too heavy to be considered for a handset and an alternative technology must be used. It has been found, however, that a parasitic PILA works well as a low band element. In this hybrid arrangement, an elevated pellet is driven from a microstrip feed and matching circuit and, in turn, the pellet feeds the PILA, as shown in figure 1.

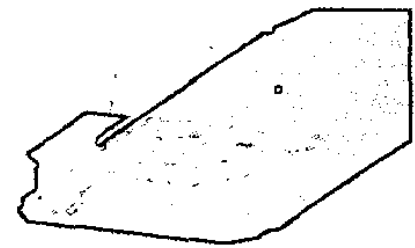

Fig. 1. View of a mobile telephone antenna with an elevated ceramic pellet dielectrically exciting a PILA passing over it. 
As figure 1 shows, the conductive direct feed structure extends from the upper surface of the PCB and directly contacts the dielectric on one side. The low band PILA shown in figure 1 is ' $S$ ' shaped and has a shorting pin close to the ceramic. However, other arrangements are possible and figure 2 shows an alternative where the PILA is a spiral shaped with an grounding pin further away from the ceramic pellet. The pellet can be fed on any of the side faces, and also on the underside using a spring-loaded "Pogo-pin", as shown in figure 2 .

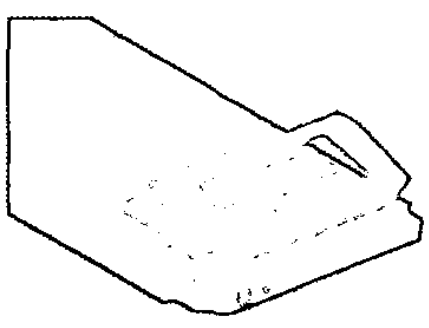

Fig. 2. View of a mobile telephone antenna with an elevated ceramic pellet fed on the underside via a spring-loaded Pogo-pin.

\section{MEASUREMENTS}

Figure 3 shows the measured return loss for a typical prototype.

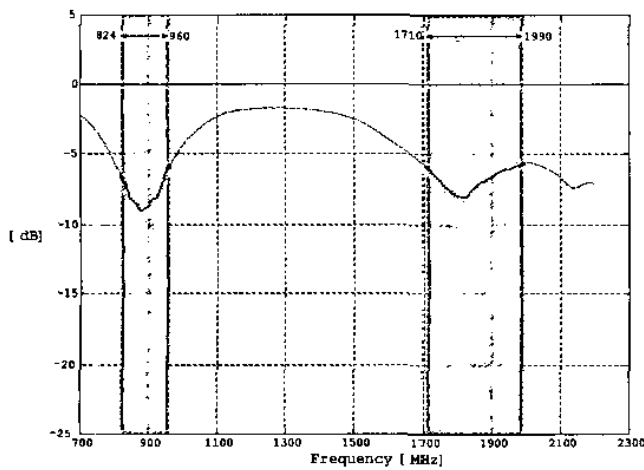

Fig. 3. Typical return loss of an elevated-pellet handset antenna.
The average low-band efficiency was found to be around $50 \%$ and the upper band near $60 \%$, on a $98 \mathrm{~mm}$ long PCB. The volume of the antenna is $3.5 \mathrm{cc}$ and the expected resistance to detuning was confirmed.

\section{FUTURE DEVELOPMENTS}

A likely requirement for future handsets is for multiple antennas such that high data rates can be achieved in the WCDMA band through the use of diversity, MIMO or similar techniques. One method of achieving this is to use multiple elevated pellets for the high band and to use just one of these to excite a parasitic low-band PILA. This idea is shown in figure 4.

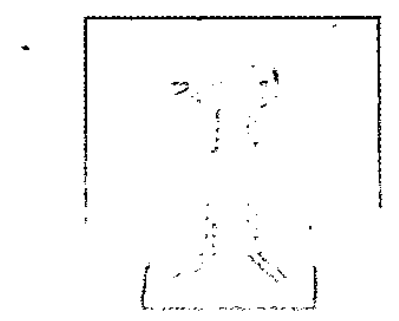

Fig. 4. MIMO handset antenna arrangement.

Several prototype boards have been built with four antennas arranged as in figure 4 on a $50 \times 100 \mathrm{~mm}$ PCB. These prototypes used the earlier type techniques of removing groundplane from under the antenna. The results showed that 3-D correlation coefficients could be obtained that were all below 0.3. This is very acceptable for MIMO or diversity applications.

\section{REFERENCES}

[1] "Advances in Antennas: The development of dielectric antenna technology", Antenna Systems 2003, Denver CO, Oct $8 / 92003$.

[2] LONG, S.A., McAlLISTER, M.W., and SHEN, L.C.: "The Resonant Cylindrical Dielectric Cavity Antenna", IEEE Transactions on Antennas and Propagation, AP-31, 1983, pp 406-412.

[3] MONGIA, R.K. and BHARTIA, P.: "Dielectric Resonator Antennas - A Review and General Design Relations for Resonant Frequency and Bandwidth", International Journal of Microwave and Millimetre-Wave Computer-Aided Engineering, 1994, 4, (3), pp 230-247. 